\title{
Pediatric Liver Transplantation, Comparative Outcomes for Patients with Biliary Atresia
}

\author{
Bruno Catoia Fonseca ${ }^{1 *}$, Gloria González García ${ }^{2}$, Mario Uribe ${ }^{1}$, Bessie Hunter ${ }^{1}$, Carolina González ${ }^{1}$, and Catalina \\ Hormazabal $^{1}$
}

${ }^{1}$ Department of Pediatric Surgery, Universidad de Chile, Chile

${ }^{2}$ Luis Calvo Mackenna Hospital, Chile

*Corresponding author: Bruno Catoia Fonseca, Medical Surgeon, Pediatric Surgery, Universidad de Chile, Campus Oriente, Santiago, Chile

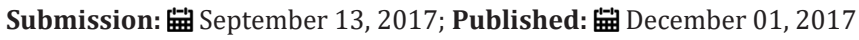

\begin{abstract}
Introduction: Orthotropic Liver Transplantation (OLT) is an effective therapeutic option to the end-stage liver disease treatment. In pediatrics, Biliary Atresia (BA) is the most frequent etiology. It has been reported that these patients would have a worse pre-transplant clinical condition, compared to other etiologies recipients; all associated with a major technical difficulty, which would result in major perioperative morbidity. Our aim is to develop an analysis of OLT due to BA outcomes versus other etiologies (Non- BA).
\end{abstract}

Materials and Methods: We retrospectively analyzed cases of transplant patients in our center between 2008 and2015. Patients were managed according to our Hospital established protocols, which provide criteria for enlistment, stay in Intensive Care Unit (ICU), diagnosis and management of complications and medical discharge. This analysis was developed to two main groups: BA and Non-BA, and also to statistically significant demographic variables subgroups: Living Donor (LD)/ Diseased Donor (DD)/ Reduced Grafts (RG)/ Complete Grafts (CG).

The statistical analysis was made with the chi-square test and the Mann-Whitney test; graft survival and patients are illustrated in Kaplan-Meier method and compared with log-rank test. The $p$ value $\leq 0.05$ was considered statistically significant.

Results: 100 OLT were carried out between 2008 and 2015. 84 patients qualified for the study, and 16 re-transplants were excluded. 43 patients (51\%) were with BA. The median age was 23 months (6-156) in BA, and 57 months (11-180) in Non-BA. The 35\% received grafts from a DD (15/43) in BA, and the $73 \%$ in Non-BA (30/41), p $\leq 0.05$, reduced grafts in 79\% (34/43) of BA and a 46.3\% in p $\leq 0.05$ (19/41). The red blood cells intra-operative use, operating time and days of stay in the ICU were similar. The Biliary and vascular complications frequency (14\% vs. $7 \%$ ) was higher in the transplant group of BA, without reaching statistical significance. In the subgroups analysis, a statistical significance was proved to the major use of red blood cells in reduced grafts $\mathrm{p} \leq 0.05$. The graft of one year and 5 years, as well as patients survival, were similar.

Conclusion: We demonstrate that the overall outcome intra-operative and postoperative in our series is similar, regardless of etiology. Although there was a higher rate of biliary complication in transplant by BA, this was not reflected in the long-term graft survival.

\section{Introduction}

Orthotropic liver transplantation (OLT) has become an effective alternative treatment of acute and chronic end-stage liver diseases. In the pediatric population, the most common cause leading to OLT is biliary atresia (BA), which outperforms universally acute liver failure, tumors, hepatitis, among others [1-4].

BA is a progressive and diffuse extra-hepatic bile duct and fibro-obliterative disease, with different commitment of intrahepatic bile duct, which despite early Kasai Technique derivation, often ends up requiring transplant [5-7] in a short or a long term. It has been reported that patients with BA would have a worse clinical condition at OLT compared to receptors for other etiologies [8] associated with a higher technical difficulty, being surgery on a previously intervened anatomy, which would produce greater perioperative morbidity [9-12].
There are few studies that compare the outcomes of liver transplantation according to etiology $[13,14]$.In a recently published series by Chung et al, in which 119 patients operated were analyzed retrospectively at a single center, it is called into question that the group of patients with BA have a worse prognosis, presenting similar results post BA transplantation, compared to other etiologies [15].

In light of discussion which that study proposed, we were interested to know our reality. The number of liver transplants performed in the Hospital Luis Calvo Mackenna reflects, to a large extent, the Chilean national reality, since it is the only national reference center in the public system. It seems relevant to analyze the comparative results of OLT by etiology, in order to inform the health team and also to give our patients and their families a predictor of postoperative evolution. 
Our aim was to compare retrospectively clinical outcomes among liver transplant recipients by secondary advanced liver disease biliary atresia, and recipients from other causes intervened in our center between 2008 and 2015.

\section{Materials and Methods}

With East Metropolitan Health Service Ethics Committee approval, a retrospective study of patients transplanted was performed in our center by the same surgical team. 84 OLT were analyzed out of a 100 total OLT made between 2008 and 2015. 16 re transplants were excluded from the study to constitute special situations of different anatomy and evolution with regard to the primary OLT.

Patients were managed according to protocols established in our hospital, which stipulate criteria for enlistment, as well as their stay in Intensive Care Unit (ICU), diagnosis and management of complications and medical discharge. All patients' parents signed an informed consent authorizing the clinical and subsequent use of data anonymously to clinical reports and research studies.

Data were obtained from the database of the Unit of Solid Organ Transplant, Dr. Luis Calvo Mackenna Hospital, which records full information on: relevant demographic background of the receiver, age, sex, weight, etiology of liver damage and the type of donor (deceased donor (DD) or living donor (LD)). It also established the graft type: Full or reduced including left side of LD or DD, left livers and PELD score ('End-stage Pediatric Liver Disease'): 4.80 [Ln serum bilirubin (mg/dL)]+18.57 [Ln INR]-6.87 [Ln albumin (g/dL)] + 4.36 $(\leq 1$ year old $)+6.67$ (stunted growth). They are described intraoperative and post-operative clinical events such as intra-operative requirement units of red blood cells and platelets, operative time, postoperative ICU stay, as well as complications of the first 90 days and graft survival and patient. The measurement of time spent in the ICU was preferred over the total time of hospitalization, as the latter increases by social factors not relevant to the clinical outcome. A descriptive statistical analysis of continuous covariates was performed to determine the measures of central tendency (median in variables that were not normally distributed and mean/ standard deviation for variables that were normally distributed) and descriptive data with related nominal frequencies variables. Statistical analysis was performed in Graph Pad PRISM 6 with the chi-square test and the Mann-Whitney $U$ test. And graft survivals for patients Kaplan-Meier curves were used and compared with log-rank test. A p $\leq 0.05$ was defined as statistically significant, with a confidence interval of $95 \%$.The analysis was performed for the two main groups: BA and Non-BA, and for subgroups of demographic variables that were statistically significant between the two study groups: frequency of LD vs. DD, including reduced graft vs. full grafts.

\section{Outcomes}

84 patients were studied. The median age was 31 months (6180), 43 male and 41 female. 43 patients (51\%) were BA, 6 of them (14\%) without prior Kasai surgery. 45 receptors received deceased donor grafts (DD, 53.6\%). The annual distribution of the series is illustrated in Figure 1, distinguishing etiologic groups. The mean follow-up time of the series was 3.5 years (6.4 months and 7.1 years) (Figure 2).

\section{8-2015: BA ETIOLOGY AND OTHERS}

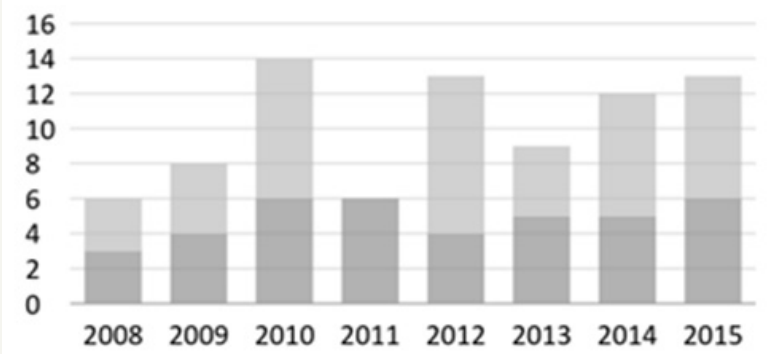

므

NON-BA

Figure 1: General Information of OLT-HLCM 2008-2015.

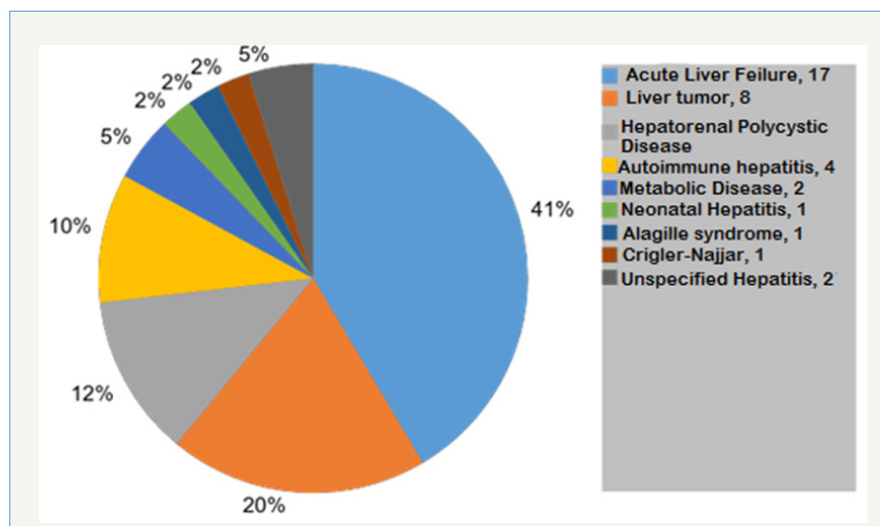

Figure 2: Etiologies that led to series of transplant OLT Non-BA.HLCM 2008-2015.

We found a PELD average score 18 (median 10), average operating time of 348 minutes (median 365) and an average of 19 days as length of stay at UCI (median 11). The survival of the entire series is illustrated in Figure 3, with values of $87 \%$ and $80 \%$ at 1 and 5 years respectively.

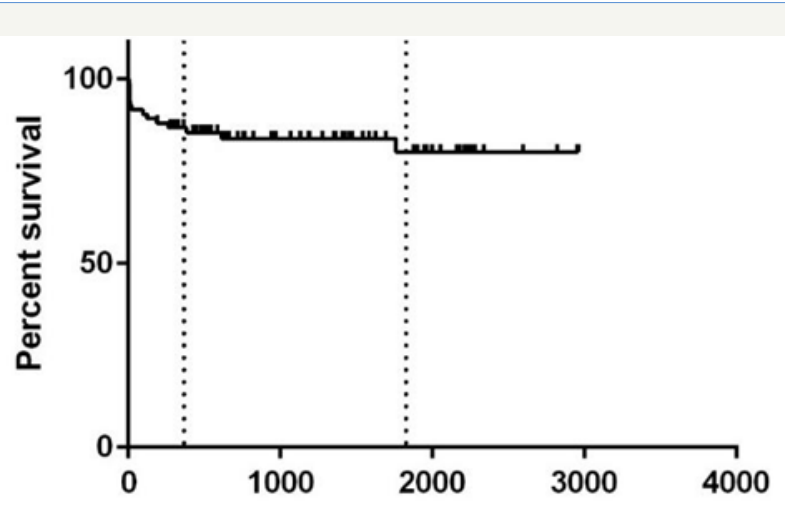

Figure 3: General Survival from Series HLCM 2008-2015. 
Graft survival at 1 and 5 years in the entire series was $85.5 \%$ and $78.8 \%$. There was a case of loss caused by a primary graft failure, within 8 days after surgery; 2 patients who died because of graft failure secondary to portal thrombosis. The other cases were loss of patient for medical reasons with a functioning graft.

\section{Comparative outcomes between BA and Non-BA groups}

Comparisons between the demographics of patients with BA $(n=43)$ and Non-BA $(n=41)$ are reported in Table 1. Patients from BA group had a median age of 23 months, versus 57 months in the
Non-BA group ( $\mathrm{p}=0.0004)$. The youngest recipient was a 6 month girl of $6 \mathrm{~kg}$, diagnosed with BA who came to transplantation without prior Kasai surgery (one of the 6/43 cases of BA group). With respect to the pre-transplant liver function in terms of PELD / MELD, no statistically significant difference was observed. As for other intra and postoperative variables, no significant differences were found between BA and Non-BA: operative time (370min vs. $380 \mathrm{~min}, \mathrm{p}=0.8350$ ), postoperative ICU stay (10days vs. 13 days, $\mathrm{p}=0.6612$ ).

Table 1: Demographic and perioperative variables: BA vs. Non-BA.

\begin{tabular}{|c|c|c|c|c|}
\hline & Variables* & BA (n= 43) & Non-BA (n=41) & P (T-Student) \\
\hline \multirow{4}{*}{ PREOP(before surgery) } & Gender (M:F) & $15: 28$ & $28: 13: 00$ & n/a \\
\cline { 2 - 5 } & Age (months) & $23(6-156)$ & $57(11-180)$ & 0.0004 \\
\cline { 2 - 5 } & Weight (kg) & $10(5.9-47.5)$ & $18.7(6.5-68.9)$ & 0.0001 \\
\hline \multirow{3}{*}{ INTRAOP(during surgery) } & PELD & $5(1-24)$ & $4(1-26)$ & 0.5843 \\
\cline { 2 - 5 } & OLT -DD & $35 \%(15 / 43)$ & $43 \%(30 / 41)$ & 0.0006 \\
\cline { 2 - 5 } & Reduced graft & $79 \%(34 / 43)$ & $5.6(1.1-23.6)$ & 0.0155 \\
\cline { 2 - 5 } & Surgical time (min) $* *$ & $6.8(0-23.6)$ & $380(210-530)$ & 0.0582 \\
\hline \multirow{3}{*}{ POSTOP (after surgery) } & Time spent in ICU (days) $* * *$ & $10(4-198)$ & $13(4-118)$ & 0.835 \\
\cline { 2 - 5 } & $\begin{array}{c}\text { Complications requiring } \\
\text { re-operation }\end{array}$ & $44 \%(19 / 43)$ & $37 \%(15 / 41)$ & 0.6612 \\
\hline
\end{tabular}

${ }^{*}$ Median values (range) is reflected

** Surgical time in patients with hepatorenal transplant is excluded

*** The period of hospitalization were excluded in ICU patients with early postoperative mortality

PELD = pediatric end-stage liver disease score; GR: Units of Red Blood Cells; ICU: Intensive Care Unit

The average use of red cell units for intra operative group BA was 6.8 against 5.6 units, $\mathrm{p}=0.0582$. BA patients received grafts DD in $35 \%$ of cases versus $73 \%$ in the group of Non-BA.

Surgical complications requiring re-intervention by surgery and/or interventional radiology are described in Table 2. The overall incidence was $40.4 \%(n=34)$, similar in both groups $(44 \%$ and $37 \%, \mathrm{p}=0.4781$ ). The overall incidence of complications related to transplantation was $35.7 \%$ (30/84). There were no significant differences in the incidence of bleeding or vascular complications between the two groups. The incidence of biliary complications (leakage and stenosis, specifically) was higher in the group of BA, although not statistically significant $(16 \%$ vs. $7 \%$, $p=0.2049)$.

Table 2: Postoperative complications: ba vs. Non-ba.

\begin{tabular}{|c|c|c|c|}
\hline Complications & BA (n= 43) & No-BA (n= 41) & P value \\
\hline Overall Reoperations & $44 \%(n=19)$ & $37 \%(n=15)$ & 0.4781 \\
\hline Hemorrhage & $5 \%(n=2)$ & $5 \%(n=2)$ & 0.9611 \\
\hline Biliary complication & $14 \%(n=6)$ & $7 \%(n=3)$ & 0.6162 \\
\hline$\rightarrow$ Biliodigestive Reanastomosis & $\rightarrow 7 \%(n=3)$ & $17 \%(n=7)$ & 0.6527 \\
\hline Vascular complications & $21 \%(n=9)$ & $\rightarrow 5 \%(n=2)$ & 1 \\
\hline$\rightarrow$ Hepatic artery thrombosis & $\rightarrow 7 \%(n=3)$ & $\rightarrow 5 \%(n=2)$ & A \\
\hline$\rightarrow$ Portal veinthrombosis & $\rightarrow 7 \%(n=3)$ & & \\
\hline
\end{tabular}


Survival curves of patients from groups BA and Non-BA are shown in Figure 4. The recipients of BA group had a year survival and at 5 years of $81.3 \%$; the group No-BA survival had a $92.5 \%$ at a year and $79.2 \%$ at 5 years ( $\mathrm{p}=0.57$ ). Graft survival of BA group was a $81.3 \%$ at a year and at 5 years; the group No-BA was $87.8 \%$ at a year, and $74.4 \%$ at 5 years $(\mathrm{p}=0.96)$.

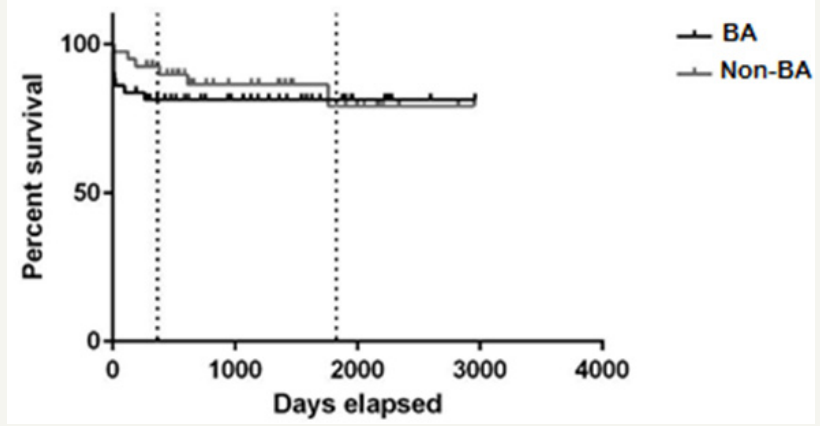

Figure 4: Comparative Survival: BA vs. Non-BA.

\section{Comparative results of DD and LD groups}

The $34.8 \%$ of transplant patients with DD were BA, while in the LD group, a $68.4 \%$ were BA. Age was significantly lower in LD receptors compared to DD (median 19 months vs. 60 months, respectively). Also, the weight of LD recipient was significantly lower compared to DD (median 10 vs. $20 \mathrm{~kg}$ ). PELD preoperative, operative time, intraoperative red blood cell consumption and time spent in the ICU were comparable between the 2 groups, with no statistically significant difference (Table 3 ).

Table 3: Demographic and perioperative variables: DD vs. LD.

\begin{tabular}{|c|c|c|c|}
\hline Variables $*$ & DD (n= 46) & LD (n= 38) & P value \\
\hline BA Percentages & $34.8 \%(16 / 46)$ & $68.4 \%(26 / 38)$ & 0.0041 \\
\hline GR use (one=10cc/kg) & $5.7(1-24)$ & $7(0-23.6)$ & 0.2555 \\
\hline Surgical time (min) $* *$ & $420(240-540)$ & $365(210-540)$ & 0.306 \\
\hline $\begin{array}{c}\text { Time spent in ICU (days) } \\
* *\end{array}$ & $11(4-198)$ & $10.5(4-118)$ & 0.1995 \\
\hline $\begin{array}{c}\text { Complications demanding } \\
\text { surgery }\end{array}$ & $33 \%(15 / 46)$ & $37 \%(14 / 38)$ & 0.8181 \\
\hline
\end{tabular}

Table 4: Postoperative complications: DD vs. LD.

\begin{tabular}{|c|c|c|c|}
\hline Complications & DD (n= 46) & LD (n= 38) & P value \\
\hline Overall Reoperations & $33 \%(n=15)$ & $37 \%(n=14)$ & 0.818 \\
\hline Hemorrhage & $4 \%(n=2)$ & $5 \%(n=2)$ & 1 \\
\hline Biliary complication & $7 \%(n=3)$ & $16 \%(n=6)$ & 0.288 \\
\hline $\begin{array}{c}\text { Reanastomosisbilio } \\
\text { digestiva }\end{array}$ & $\rightarrow 2 \%(n=1)$ & $\rightarrow 8 \%(n=3)$ & 0.324 \\
\hline Vascular complications & $22 \%(n=10)$ & $16 \%(n=6)$ & 0.583 \\
\hline Hepatic artery thrombosis & $\rightarrow 7 \%(n=3)$ & $\rightarrow 5 \%(n=2)$ & 1 \\
\hline$\rightarrow$ Portal vein thrombosis & $\rightarrow 7 \%(n=3)$ & $\rightarrow 3 \%(n=1)$ & 0.623 \\
\hline
\end{tabular}

The presentation of complications demanding re-intervention by surgery and / or interventional radiology, are comparatively listed in Table 4: the overall incidence was similar between DD and $\mathrm{LD}(33 \%$ and $37 \%$, respectively, $\mathrm{p}=0.8181$.). The incidence of biliary complications was higher in LD ( $16 \%$ vs. $7 \%, p=0.288)$, while vascular complications, including thrombosis, occurred more frequently in DD (22\% vs. 16\%, p=0.5825), without achieving any demonstrated significance of these differences.

DD receivers had a survival at a year and at 5years of $84.3 \%$ and $77.8 \%$, respectively; the LD group had a survival rate of $89.5 \%$ at a year and $83.2 \%$ at 5years ( $\mathrm{p}=0.805)$. The graft survival of LD group was $89.5 \%$ at a year, and at 5years was $83.2 \%$; DF group had a survival of $80.4 \%$ at a year and $73.7 \%$ at 5years $(p=0.47)$

\section{Comparative outcomes between Complete Graft (CG) vs. Reduced Graft (RG)}

Complete grafts were transplanted in 28 patients and Reduced Grafts in 56 patients. These grafts concerned BA in a $32.1 \%$ and $60.7 \%$ respectively. Age was significantly lower in RG receivers with regard to CG (median 23months vs. 113months, respectively) and weight (median 10.8 vs. 29kg). Unlike previously compared groups, a difference was observed in the intraoperative consumption of red blood cells according to the type of transplanted graft, being major in the Reduced Graft group (7 vs. 3.4 units $10 \mathrm{cc} / \mathrm{kg}$ ). Preoperative PELD, operative time and length of stay in the ICU were comparable between the 2 groups, with no statistically significant difference (Table 5).

Table 5: Demographic and perioperative variables: CG vs. RG.

\begin{tabular}{|c|c|c|c|}
\hline Variables * & Complete (n= 28) & Reduced (n= 56) & P value \\
\hline BA Percentages & $32.1 \%(9 / 28)$ & $60.7 \%(34 / 56)$ & 0.0202 \\
\hline $\begin{array}{c}\text { GR use (one=10cc/ } \\
\text { kg) }\end{array}$ & $3.4(1,1-9,4)$ & $7(0-23.6)$ & $<0.0001$ \\
\hline $\begin{array}{c}\text { Surgical time (min) } \\
* *\end{array}$ & $360(240-510)$ & $390(210-540)$ & 0.953 \\
\hline $\begin{array}{c}\text { Time spent in ICU } \\
\text { (days) } * *\end{array}$ & $10(4-34)$ & $11(4-198)$ & 0.126 \\
\hline $\begin{array}{c}\text { Complications } \\
\text { demanding } \\
\text { surgery }\end{array}$ & $36 \%(10 / 28)$ & $34 \%(19 / 56)$ & 0.488 \\
\hline
\end{tabular}

* Average values (range) is considered

** The period of hospitalization is excluded in ICU patients with early postoperative mortality.

PELD: Pediatric end-stage liver disease score; GR: Units of Red Blood Cells; ICU: Intensive Care Unit.

The presentation of complications requiring surgical reintervention or interventional radiology is comparatively listed in Table 6: the overall incidence was similar between CG and RG (36\% and $34 \%$, respectively). The incidence of biliary complications was higher in RG (14\% vs. $4 \%$ ), while vascular, including real thrombosis, occurred more frequently in CG (25\% vs. 16\%), without significance in any of those differences. 
Table 6: Postoperative complications: Complete Graft vs. Reduced graft.

\begin{tabular}{|c|c|c|c|}
\hline Complications & Complete $(\mathbf{n = 2 8 )}$ & Reduced (n= 56) & P Value \\
\hline Overall Reoperations & $36 \%(\mathrm{n}=10 / 28)$ & $34 \%(\mathrm{n}=19 / 56)$ & 0.488 \\
\hline Hemorrhage & $7 \%(\mathrm{n}=2)$ & $4 \%(\mathrm{n}=2)$ & 0.598 \\
\hline Biliary complication & $4 \%(\mathrm{n}=1)$ & $14 \%(\mathrm{n}=8)$ & 1 \\
\hline$\rightarrow$ Reanastomosisbilio digestiva & $\rightarrow 4 \%(\mathrm{n}=1)$ & $\rightarrow 5 \%(\mathrm{n}=3)$ & 0.382 \\
\hline Vascular complications & $25 \%(\mathrm{n}=7)$ & $\rightarrow 5 \%(\mathrm{n}=9)$ & 1 \\
\hline$\rightarrow$ Hepatic artery thrombosis & $\rightarrow 7 \%(\mathrm{n}=2)$ & $\rightarrow 5 \%(\mathrm{n}=3)$ & 1 \\
\hline
\end{tabular}

Complete graft recipients had a survival at a year and at 5 years of $87.4 \%$; reduced graft recipients had a survival of $85.5 \%$ at a year and $81.1 \%$ at 5 years $(p=0.472)$. Complete graft survival was $89.1 \%$ at a year and at 5 years was $78 \%$; also, reduced graft survival was $82.1 \%$ at a year and $77.8 \%$ at 5 years $(p=0.40)$.

\section{Discussion}

BA is still a treatment challenge. It is expected that $60-80 \%$ of patients, despite optimal management and timely biliodigestive bypass, will have chronic irreversible changes in their native liver, requiring liver transplantation in a follow-up of 20 years [16,17]. In addition, the late referral for study and portoenterostomy remains a significant aggravating factor in our median: the average age of patients at the time of referral biliodigestive in this series is 72 days. Thus, liver transplantation in pediatric age takes an important role in the management of Biliary Atresia (BA).

Since our first surgery case in 1996, they have been completed to date 219 pediatric liver transplants in Luis Calvo Mackenna Hospital, national reference center in the public system for this procedure. The figure includes DD and LD liver transplants. The Hospital has implemented a transplant unit for solid organs and a multidisciplinary unit for optimal management of these patients, considering that in the past this was conducted on a small scale. Our outcomes are now comparable with those of the international literature [18].

Starlz and Gordon reported in 1985 at the American College of Surgeons that OLT was noticeably more difficult in patients with prior Kasai surgery [19]. Despite this real technical difference, postoperative results have shown to be comparable. The results of OLT in BA with and without prior Kasai are similar, despite the recipient's weight, which has been the reason of the use of "primary" OLT as an alternative in selected cases that clinically could not benefit from portoenterostomy [20,21].

We confirmed that these patients have a weight and age significantly smaller compared with group of patients transplanted for other etiologies, when comparing the BA transplanted of our series. This leads to an increased use of small grafts and live donors in this group, which it would be linked to an increased frequency of both vascular and biliary complications after surgery $[22,23]$.
It is difficult to compare the pre-transplant clinical condition, considering etiological characteristics of the group No-BA. The PELD score, designed to categorize pediatric patients with chronic liver damage, being transplant candidates, was the most likely parameter to contrast the studied groups. Still, it does not provide a true reflection of acute liver dysfunction (e.g. AHF: 'acute hepatic failure'). In other situations, including some metabolic diseases and in most tumors, it is expected that patients do not show liver dysfunction, so PELD score is not a reliable parameter. In our experience with BA patients were transplanted with an average score of 9.

When analyzing the intraoperative behavior, a major requirement of blood transfusion was observed in the BA group, which is opposite to the belief of a greater intra-operative bleeding associated with reoperation; however the blood transfusion requirement was significantly higher when reduced graft from DD was used, versus complete graft or graft from living donors (LD). This is explained by the liver transection, ex-vivo performed, in this type of graft. Other perioperative parameters were not significant between analyzed groups, similar to that described in the series of Chung et al.

Graft survival was affected neither by etiology, nor by the use of grafts from living donors. Also, it is not affected by the use of reduced graft. The highest percentage of graft loss in the series was due to the death for medical reasons, similar to the group of BA and Non-BA.

Overall survival of the series resembles the expected actuarial survival according to world literature. In comparative analysis, no significant difference was observed in terms of patient survival. As expected, most patients' loss occurs during the first year of post-transplant, due to mainly infectious medical complications. Note that in the BA group, there were: $2 / 8$ patients who died of graft failure secondary to portal thrombosis, $1 / 8$ by primary graft failure and other patients for sepsis. In the group Non-BA, mortality was due to medical reasons. The greater frequency belongs to the transplantation for liver tumors 4/8, with: 1 death from recurrence of disease after lung transplantation, 1 patient with post-transplant lymphoma who did not respond to chemotherapy, 1 sepsis and 1 patient for infection parvovirus B19, at 4 years post-transplant. 


\section{Conclusion}

We reassure that the intra- and postoperative overall results of our series are similar, regardless of the etiology that leads to transplantation. Although there is a higher rate of biliary complications, these have not been reflected in long-term graft survival.

\section{References}

1. Pérez R, Sanhueza E, Hunter B, et al. (2010) Capítulo IX: Trasplante hepático. En: Guías Clínicas - Sociedad Chilena de Trasplante. Uribe M editor Santiago, Chile.

2. Tannuri AC, Gibelli NE, Ricardi LR, Silva MM, Santos MM, et al. (2011) Orthotopic liver transplantation in biliary atresia: a single-center experience. Transplant Proc 43(1): 181-183.

3. Migliazza L, Lopez Santamaria M, Murcia J, Gamez M, Clavijo J, et al. (2000) Long-term survival expectancy after liver transplantation in children. J PediatrSurg 35(1): 5-7.

4. Goss JA, Shackleton CR, Swenson K, Satou NL, Nuesse BJ, et al. (1996) Orthotopic liver transplantation for congenital biliary atresia. An 11year, single-center experience. Ann Surg 224(3): 276-284.

5. Visser BC, Suh I, Hirose S, Rosenthal P, Lee H, et al. (2004) The influence of portoenterostomy on transplantation for biliary atresia. Liver Transpl 10(10): 1279-1286.

6. Davenport M (2012) Biliary atresia: clinical aspects. Semin Pediatr Surg 21(3): 175-184.

7. Lee V, Haber B, Magee J, Miethke A, Murray KF, et al. (2014) Medical Status of 219 Children with Biliary Atresia Surviving Long-Term with Their Native Livers: Results from a North American Multicenter Consortium. J Pediatr 165(3): 539-546.

8. Barshes NR, Chang IF, Karpen SJ, Carter BA, Goss JA, et al. (2006) Impact of pretransplant growth retardation in pediatric liver transplantation. J Pediatr Gastroenterol Nutr 43(1): 89-94.

9. Utterson EC, Shepherd RW, Sokol RJ, Bucuvalas J, Magee JC, et al. (2005) Biliary atresia: clinical profiles, risk factors, and outcomes of 755 patients listed for liver transplantation. J Pediatr 147(2): 180-185.

10. Takahashi Y, Nishimoto Y, Matsuura T, Hayashida M, Tajiri T, et al. (2009) Surgical complications after living donor liver transplantation in patients with biliary atresia: a relatively high incidence of portal vein complications. Pediat rSurg Int 25(9): 745-751.
11. Li-Ying Sun, Yun-Sheng Yang, Zhi-Jun Zhu, Gao W, Wei L, et al. (2013) Outcomes in children with biliary atresia following liver transplantation. Hepatobiliary Pancreat Dis Int 12(2): 143-148.

12. Anderson CD, Turmelle YP, Lowell JA, Nadler M, Millis M, et al. (2008) The effect of recipient-specific surgical issues on outcome of liver transplantation in biliary atresia. Am J Transplant 8(6): 1197-204.

13. Cowles R, Lobritto S, Ventura K, Harren PA, Gelbard R, et al. (2008) Timing of liver transplantation in biliary atresia results in 71 children managed by a multidisciplinary team. J Pediatr Surg 43(9): 1605-1609.

14. Mizuta K, Sanada Y, Wakiya T, Urahashi T, Umehara M, et al. (2010) Living Donor Liver Transplantation in 126 Patients with Biliary Atresia: SingleCenter Experience. Transplant Proc 42(10): 4127-4231.

15. Chung PH, Wong KK, Chan SC, Tam PK (2015) Liver transplant for biliary atresia is associated with a worse outcome - Myth or fact? J Pediatr Surg 50(12): 2134-2136.

16. Shinkai M, Ohhama Y, Take H, Kitagawa N, Kudo H, et al. (2009) Longterm outcome of children with biliary atresia who were not transplanted after the Kasai operation: >20-year experience at a children's hospital. J Pediatr Gastroenterol Nutr 48(4): 443-450.

17. de Vries W, Homan-Van derVeen J, Hulscher JB, Hoekstra-Weebers JE, Houwen RH, et al. (2011) Twenty-year transplant-free survival rate among patients with biliary atresia. Clin Gastroenterol Hepatol 9(12): 1086-1091.

18. Acuña C, Zuleta R, Dalmazzo R, Valverde C, Uribe M, et al. (2013) Pediatric liver transplantation experience and outcome in Chile. Transplant Proc 45(10): 3724-3725.

19. Starzl TE, Gordon RD (1985) Liver transplantation in children-A solution for biliary atresia? Proc Am Co11 Sur 71: 17-18.

20. Sandler AD, Azarow KS, Superina RA (1997) The impact of a previous Kasai procedure on liver transplantation for biliary atresia. J Pediatr Surg 32(3): 416-419.

21. Safwan M, Ramachandran P, Reddy MS, Shanmugam N, Rela M, et al. (2016) Living donor liver transplantation for biliary atresia - An Indian experience. Pediatr Transplant 20(8): 1045-1050.

22. Ye H, Zhao Q, Wang Y, Wang D, Zheng Z, et al. (2015) Outcomes of Technical Variant Liver Transplantation versus Whole Liver Transplantation for Pediatric Patients: A Meta-Analysis. PLoS One 10(9).

23. Arnon R, Annunziato RA, D'Amelio G, Chu J, Shneider BL (2016) Liver Transplantation for Biliary Atresia: Is There a Difference in Outcome for Infants? J Pediatr Gastroenterol Nutr 62(2): 220-225. 